Research Paper

\title{
The Clinical Impact of c-MET Over-Expression in Advanced Biliary Tract Cancer (BTC)
}

\author{
Mi Hwa Heo ${ }^{1}$, Hee Kyung Kim¹, Hansang Lee ${ }^{1}$, Kyoung-Mee Kim², Jeeyun Lee', Se Hoon Park ${ }^{1}$, Joon Oh \\ Park ${ }^{1}$, Ho Yeong Lim ${ }^{1}$, Won Ki Kang ${ }^{1}$, Young Suk Park ${ }^{1}$, and Seung Tae Kim${ }^{1 凶}$ \\ 1. Division of Hematology-Oncology, Department of Medicine, Samsung Medical Center, Sungkyunkwan University School of Medicine, Seoul, Korea; \\ 2. Department of Pathology \& Translational Genomics, Samsung Medical Center, Sungkyunkwan University School of Medicine, Seoul, Korea. \\ $\square$ Corresponding author: Seung Tae Kim, MD, PhD., Division of Hematology-Oncology, Department of Medicine, Samsung Medical Center, Sungkyunkwan \\ University School of Medicine, 81 Irwon-ro, Gangnam-gu, Seoul 135-710, Korea Tel: +82-2-3410-0297 Fax: +82-2-3410-1754 E-mail: seungtae1.kim@samsung.com \\ (c) Ivyspring International Publisher. This is an open access article distributed under the terms of the Creative Commons Attribution (CC BY-NC) license \\ (https://creativecommons.org/licenses/by-nc/4.0/). See http://ivyspring.com/terms for full terms and conditions.
}

Received: 2016.10.13; Accepted: 2017.03.21; Published: 2017.05.12

\begin{abstract}
Background: c-MET is a proto-oncogene that encodes the tyrosine kinase receptor for hepatocyte growth factor (HGF). Activation of HGF-c-MET signaling involves cell invasiveness and evokes metastasis through direct involvement of tumor angiogenesis. However, the value of c-MET overexpression is still unknown in metastatic biliary tract cancer (BTC).

Methods: We analyzed the incidence and clinicopathologic characteristics of c-MET overexpression in advanced BTC. Moreover, we investigated the value of c-MET overexpression in predicting response to gemicitabine plus cisplatin (GC), a first line standard regimen, and as a prognostic marker in metastatic BTC.

Results: The BTC subtype distribution ( $N=44$ ) was as follows: intrahepatic cholangiocarcinoma (IHCC, $n=7)$, extrahepatic cholangiocarcinoma (EHCC, $n=25)$ and gallbladder cancer $(\mathrm{GBC}, \mathrm{n}=12)$. Liver $(52.3 \%)$ was the predominant metastatic site, followed by lymph nodes (36.4\%) and bone (15.9\%). Among the 44 patients analyzed for c-MET expression, 15 (34.1\%) exhibited c-MET overexpression in tumor tissues. There was no significant difference in the prevalence of c-MET overexpression among primary sites in EHCC $(7 / 25,28.0 \%)$, IHCC (3/7, 42.9\%), and GBC (5/12, 41.7\%). There was also no significant correlation between specific clinicopathologic variables and c-MET expression. Comparing the tumor-response to GC according to c-MET expression (overexpression vs. non-overexpression), there was no significant difference in either RR or DCR ( $p=0.394$ and $p>0.999$, respectively). The median PFS for all 44 patients was 9.00 months $(95 \% \mathrm{Cl}, 7.5-10.5$ months) and there was no significant difference for PFS between patients with c-MET overexpression and those without $(p=0.917)$. The median OS was 14.4 months $(95 \% \mathrm{Cl}, 11.9-16.9$ months $)$. There was no significant difference in OS between patients with c-MET overexpression compared to those without (13.7 vs. 14.4 months, respectively; $p=0.708$ ).

Conclusions: c-MET overexpression was detected in $34.1 \%$ of advanced BTC patients irrespective of tumor location. c-MET overexpression did not predict response to GC or survival. Further studies are needed to fully elucidate the value of c-MET overexpression as a novel biomarker in these patients.
\end{abstract}

Key words: c-MET, Biliary tract cancer (BTC).

\section{Introduction}

Biliary tract cancer (BTC) is an aggressive disease with a very poor prognosis and a median survival of less than one year [1]. It is a heterogeneous group of diseases including intrahepatic, perihilar, or distal cholangiocarcinoma and gallbladder cancer, with diverse epidemiology, etiology, and pathogenesis. Personalized medicine is defined as the use of an individual patient's molecular information to inform diagnosis, prognosis, treatment, and prevention of cancer, and has become a primary focus of many studies in oncology [2]. Indeed, the identification of genomic alterations associated with responses to molecularly targeted agents, such as tyrosine kinase inhibitors, has changed the paradigm of cancer treatment into precision medicine by identification of multiple actionable targets across cancer types, 
especially with the emergence of advanced genomic techniques [3, 4]. Thus, it is necessary to improve our understanding of the heterogeneity of disease at the genomic and molecular levels. However, the molecular and genetic features of BTC have been inadequately investigated in comparison to other common solid cancers. Recently, it has been proposed that receptor tyrosine kinases such as epidermal growth factor receptor, vascular epithelial growth factor receptor, and c-MET are promising targets for cholangiocarcinoma [5-7].

c-MET is a proto-oncogene that encodes the tyrosine kinase receptor for hepatocyte growth factor (HGF). Activation of HGF-c-MET signaling involves cell invasiveness and evokes metastasis through direct involvement of tumor angiogenesis [8, 9]. Enhanced expression of the c-MET protein has been reported in various solid cancers such as breast cancer [10], gastric cancer [11], lung cancer [12, 13] and hepatocellular carcinoma [14, 15]. c-MET overexpression has been detected in $11.7 \%$ and $16.2 \%$ of intra-and extra-hepatic cholangiocarcinoma cases, respectively, and is associated with inferior post-resection recurrence-free survival [16]. However, c-MET expression was $76.7 \%$ and did not play a prognostic role in extra-hepatic cholangiocarcinoma patients with curative resection followed by adjuvant chemo-radiotherapy [17]. The value of c-MET overexpression remains unknown in metastatic BTC.

Herein, we report the incidence and clinicopathologic characteristics of advanced BTC with and without c-MET overexpression. Moreover, we investigated the value of c-MET overexpression in predicting response to gemicitabine plus cisplatin (GC), a first line standard regimen, and as a prognostic marker in metastatic BTC.

\section{Materials and Methods}

\section{Patients}

We analyzed the expression of c-MET proteins using immunohistochemistry (IHC) in tumor samples of metastatic BTC. Tumor tissues originated from 44 patients who underwent palliative gemcitabine plus platinum chemotherapy as first line therapy at Samsung Medical Center between January 2012 and March 2016. Clinical data were retrospectively obtained from electronic medical records and included sex, age at diagnosis, subtypes of BTC, pathologic profiles (tumor size, differentiation, margin status, lymphovascular invasion, perineural invasion, and vessel involvement), extent of metastasis, regimen for chemotherapy, tumor response and the date of disease-progression or death.

\section{Immunohistochemistry}

IHC was performed for c-MET. Hematoxylin-eosin stained slides were reviewed and representative formalin-fixed, paraffin-embedded archival blocks were selected for each case. MET IHC was performed using the rabbit monoclonal primary antibody CONFIRM anti-total MET (SP44) (Ventana Medical Systems, Tucson, AZ, USA) and the Ventana BenchMark XT automated slide processing system (Ventana Medical Systems) according to the manufacturer's protocol. All stained specimens were independently reviewed by a pathologist without prior knowledge of clinical information. The percentage and intensity of positive tumor cells were recorded by manually counting representative fields of each case. For MET, we applied a recently developed scoring system for gastric cancer [18]. Overexpression was defined as $2+$ or $3+$ with two pathologists in consensus.

\section{Statistics}

Descriptive statistics were reported as proportions and medians. Correlations between c-MET expression and clinicopathologic variables were analyzed using the t-test or Fisher's exact test or by one-way analysis of variance, as appropriate. Treatment outcomes were response rate (RR) and progression-free survival (PFS). Kaplan-Meier estimates were used for the analysis of all time-to-event variables and the 95\% confidence interval (CI) for the median time to event was computed. The overall survival (OS) was measured from the date of chemotherapy to the date of death from any cause and was censored at the date of the last follow-up visit. PFS was calculated from the date of chemotherapy to date of disease progression or death from any cause or the date of last follow-up. Variables with $\mathrm{p}<0.05$ were considered significant for the analysis, and $95 \%$ CI were calculated. All statistical analyses were performed using PASW Statistics version 23.0 (SPSS Inc., Chicago, IL, USA).

\section{Results}

\section{Clinical characteristics of patients}

The clinical characteristics of the advanced BTC patients in the present study are summarized in Table 1. The median age of all patients was 60.5 years (range, 37-77 years), and $30(70.2 \%)$ patients were male. The BTC subtype distribution was as follows: intrahepatic cholangiocarcinoma (IHCC, n=7), extrahepatic cholangiocarcinoma $(\mathrm{EHCC}, \mathrm{n}=25)$ and gallbladder cancer $(\mathrm{GBC}, \mathrm{n}=12)$. Liver $(52.3 \%)$ was a predominant metastatic site, followed by lymph nodes $(36.4 \%)$ and bones $(15.9 \%)$. 
Table 1. Baseline characteristics of 44 advanced BTC patients according to c-MET expression

\begin{tabular}{|c|c|c|c|c|}
\hline & & c-MET & & \\
\hline & All & $\begin{array}{l}\text { Non-overexpressio } \\
\mathrm{n}\end{array}$ & $\begin{array}{l}\text { Overexpressio } \\
\text { n }\end{array}$ & $\begin{array}{l}\text { P-valu } \\
\text { e }\end{array}$ \\
\hline Sex & & & & 0.501 \\
\hline Male & 30 & 21 & 9 & \\
\hline Female & 14 & 8 & 6 & \\
\hline Age median 60 & 7-77) years & & & 0.722 \\
\hline$\geq 65$ & 11 & 8 & 3 & \\
\hline$<65$ & 33 & 21 & 12 & \\
\hline Subtypes & & & & 0.375 \\
\hline IHCC & 7 & 4 & 3 & \\
\hline $\mathrm{EHCC}$ & 25 & 18 & 7 & \\
\hline GBC & 12 & 7 & 5 & \\
\hline Differentiatio & & & & 0.3 \\
\hline Well & 6 & 5 & 1 & \\
\hline Moderate & 26 & 17 & 9 & \\
\hline Poor & 9 & 4 & 5 & \\
\hline $\mathrm{NE}$ & 3 & 3 & 0 & \\
\hline Sites of metas & & & & 0.717 \\
\hline Liver & 23 & 17 & 6 & \\
\hline Lymph node & 16 & 10 & 6 & \\
\hline $\begin{array}{l}\text { Other organs } \\
\text { (lung, } \\
\text { bone, } \\
\text { peritoneum) }\end{array}$ & 12 & 8 & 4 & \\
\hline
\end{tabular}

\section{Correlation between c-MET overexpression and clinical variables}

Among the 44 patients analyzed for c-MET expression, 15 (34.1\%) exhibited c-MET overexpression in tumor tissues. There was no significant difference in the prevalence of c-MET overexpression among primary sites of $\operatorname{EHCC}(7 / 25$, $28.0 \%)$, IHCC $(3 / 7,42.9 \%)$, and GBC $(5 / 12,41.7 \%)$. There was also no significant correlation between specific clinicopathologic variables and c-MET expression.

\section{The impact of c-MET overexpression on treatment outcome}

All analyzed patients received gemcitabine plus platinum chemotherapy as a first line treatment, with a median cycle number of 7.5 (range 1 to 43) cycles. Six patients with partial responses were observed, revealing a RR of $13.6 \%$. Stable disease was observed in 8 patients $(18.2 \%)$. The disease control rate (DCR) was $31.8 \%$ (Table 2). Comparing the tumor-response for gemcitabine plus platinum according to c-MET expression (overexpression vs. non-overexpression), there was no significant difference for both RR and DCR $(p=0.394$ and $p>0.999$, respectively). The median PFS for all 44 patients was 9.00 months $(95 \% \mathrm{CI}$, 7.5-10.5 months) and there was no significant difference for PFS between patients with c-MET overexpression and those without ( $p=0.917)$ (Fig 1).
Table 2. Tumor response according to c-MET expression

\begin{tabular}{lllll}
\hline & & $\begin{array}{l}\text { c-MET } \\
\text { overexpression }\end{array}$ & $\begin{array}{l}\text { c-MET } \\
\text { non-overexpression }\end{array}$ \\
\hline $\begin{array}{l}\text { Gemcitabine } \\
\text { plus platinum } \\
\text { as first line } \\
\text { treatment }\end{array}$ & $\begin{array}{l}\text { Complete } \\
\text { Response }\end{array}$ & 0 & 0 & \\
& $\begin{array}{l}\text { Partial } \\
\text { Response } \\
\text { Stable Disease }\end{array}$ & 3 & 3 & \\
& $\begin{array}{l}\text { Progressive } \\
\text { Disease }\end{array}$ & 10 & 6 & \\
& $\begin{array}{l}\text { Response Rate } \\
\text { Disease control 33.3\% } \\
\text { rate }\end{array}$ & 20 & 0.394 \\
& & $31.0 \%$ & 1.000 \\
\hline
\end{tabular}

\section{(A)}

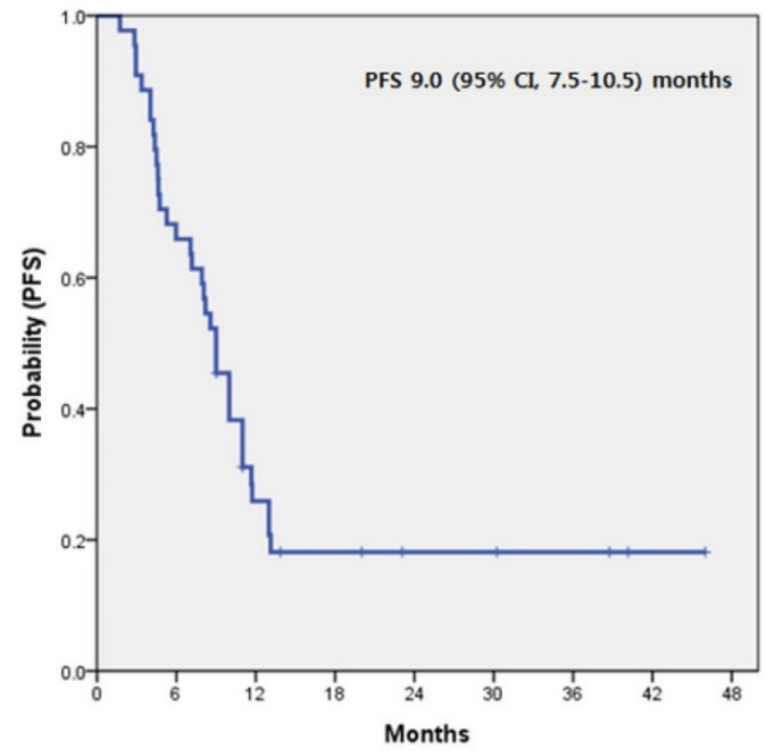

(B)

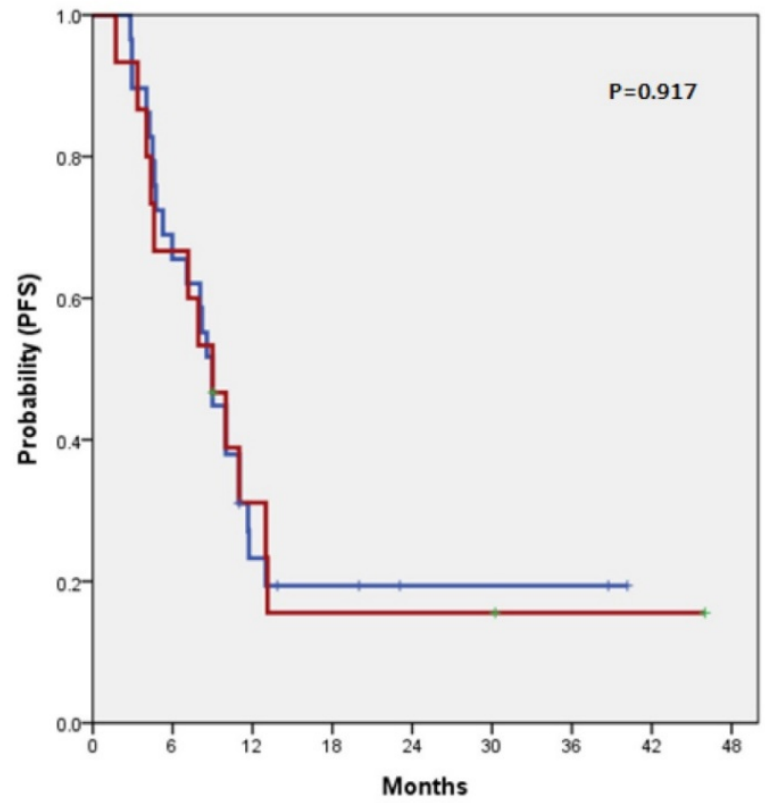

Figure 1. (A) Kaplan-Meier curve of PFS in 44 advanced BTC (B) with and without c-MET overexpression 


\section{c-MET overexpression as a prognostic factor}

The median OS was 14.4 months (95\% CI, 11.9-16.9 months). There was no a significant difference in OS between patients with c-MET overexpression and those without (13.7 vs. 14.4 months, respectively; $\mathrm{p}=0.708$ ) (Fig 2).

(A)

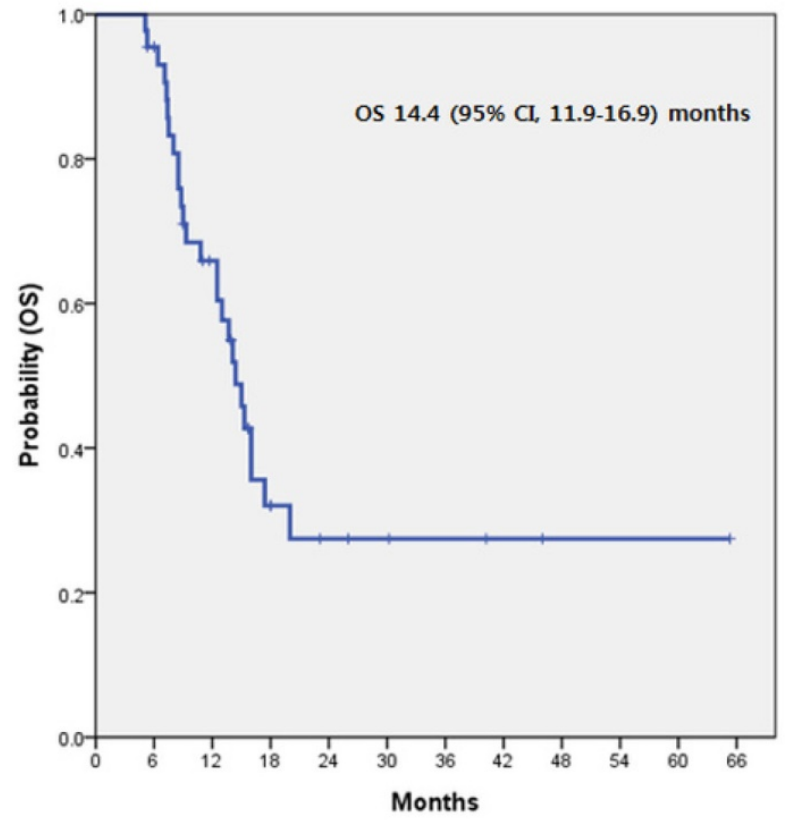

(B)

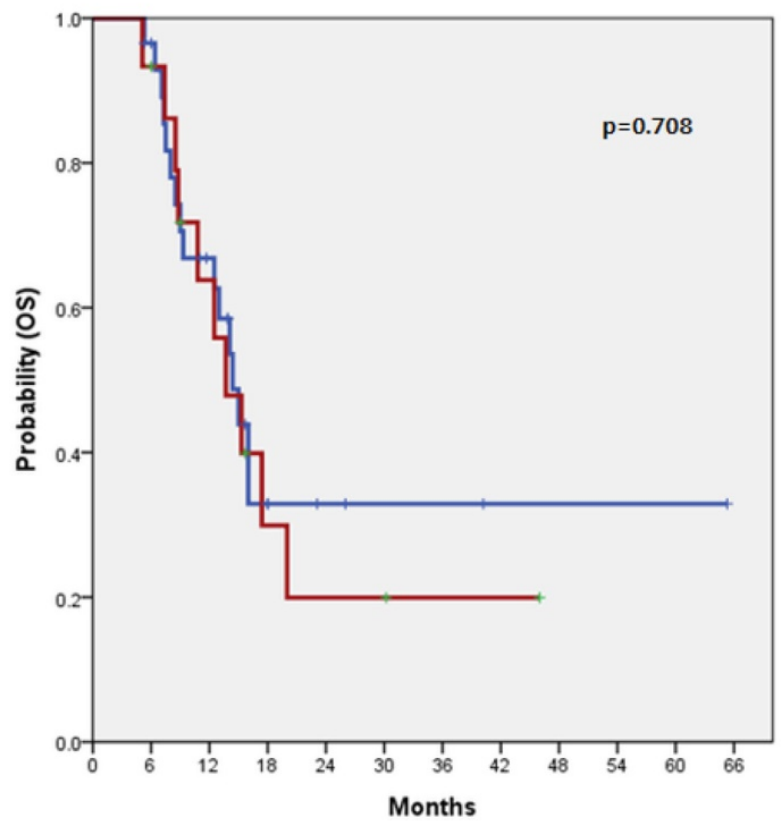

Figure 2. (A) Kaplan-Meier curve of OS in 44 advanced BTC (B) with and without c-MET overexpression

\section{Discussion}

In the present study, we analyzed the expression of c-MET in advanced BTC patients undergoing palliative chemotherapy. We also investigated the value of c-MET overexpression in predicting treatment response and prognosis for advanced BTC. Fifteen of 44 patients (34.1\%) exhibited c-MET overexpression in tumor tissues. There was no significant difference in the prevalence of c-MET overexpression among primary tumor locations. Moreover, c-MET expression was not correlated with treatment outcome and prognosis.

Previous studies of c-MET expression in BTC report positivity ranging from $0-68 \%$ [7, 16, 19-22]. The variation in these findings results from differences in the definition of c-MET overexpression, heterogeneous patient populations, and the small numbers of cases included. A standard scoring system for IHC of c-MET has not been defined. Herein, we applied a recently developed scoring system for MET IHC [18]. In our previous studies, we demonstrated that MET gene expression level correlates closely with protein overexpression when assessed using IHC. Moreover, we reported a high concordance between MET IHC assessment and NanoString-based multigene assays including MET [23]. Thus, the MET IHC method used in the present study is considered a reasonable assay for c-MET overexpression.

Miyamoto et al. examined the expression of MET and demonstrated its prognostic impact in patients with cholangiocarcinoma [16]. Positive MET expression is significantly associated with poor prognosis in patients with IHCC, but not EHCC. Multiple studies have suggested that MET overexpression is associated with poor survival in various cancers, including IHCC. In the current study, c-MET overexpression did not predict survival outcome. This discrepancy might be caused by heterogeneous patient characteristics. All patients in this study had advanced BTC. However, previous studies have included BTC patients with curative resection $[16,17]$. This difference in aggressiveness and advanced disease status would affect the prevalence and prognostic value of c-MET overexpression. Meanwhile, c-MET overexpression could be a potential therapeutic indicator to treat with c-MET pathway antagonist. However, there is few data of c-MET antagonists tested in biliary tract cancer. Recently, Barat $S$ et al. reported potent anti-tumor activity of LY2801653, a small molecule inhibitor with potent activity against MET kinase, in xenograft model using human intra- and extrahepatic cholangiocarcinoma cell lines [24]. Future, clinical trials for MET inhibitor in BTC needs to be conducted. 
The present study has some limitations, including a small sample size, retrospective nature and heterogeneous patient population. These limitations might affect the findings in this study. OS in our study seemed to be slightly longer as compared to those of previous studies [25]. Thus, findings from this study should be interpreted with caution. In spite of several limitations, this study has several strengths. We applied a reasonable IHC method to evaluate c-MET overexpression and included only advanced BTC patients, who are for a clinical unmet need.

In conclusion, c-MET overexpression was detected in $34.1 \%$ of advanced BTC patients irrespective of tumor location and did not predict response to gemcitabine plus platinum or survival. Further studies are needed to fully elucidate the value of c-MET overexpression as a novel biomarker in these patients.

\section{Acknowledgements}

This work was supported by funding from the Korean Health Technology R\&D Project, Ministry of Health \& Welfare, Republic of Korea (HI14C2750, HI14C3418). Support was also provided by a grant from Samsung Medical Center (SMX1161251).

\section{Competing Interests}

The authors have declared that no competing interest exists.

\section{References}

1. Khan SA, Taylor-Robinson SD, Toledano MB, Beck A, Elliott P, Thomas HC. Changing international trends in mortality rates for liver, biliary and pancreatic tumours. Journal of hepatology. 2002; 37: 806-13.

2. Garraway LA, Verweij J, Ballman KV. Precision oncology: an overview. Journal of clinical oncology : official journal of the American Society of Clinical Oncology. 2013; 31: 1803-5.

3. Von Hoff DD, Stephenson JJ, Jr., Rosen P, Loesch DM, Borad MJ, Anthony S, et al. Pilot study using molecular profiling of patients' tumors to find potential targets and select treatments for their refractory cancers. Journal of clinical oncology : official journal of the American Society of Clinical Oncology. 2010; 28: 4877-83.

4. Kruglyak KM, Lin E, Ong FS. Next-generation sequencing in precision oncology: challenges and opportunities. Expert review of molecular diagnostics. 2014; 14: 635-7.

5. Socoteanu MP, Mott F, Alpini G, Frankel AE. c-Met targeted therapy of cholangiocarcinoma. World journal of gastroenterology. 2008; 14: 2990-4

6. Yoshikawa D, Ojima H, Iwasaki M, Hiraoka N, Kosuge T, Kasai S, et al Clinicopathological and prognostic significance of EGFR, VEGF, and HER2 expression in cholangiocarcinoma. British journal of cancer. 2008; 98: 418-25.

7. Huang XY, Ke AW, Shi GM, Ding ZB, Devbhandari RP, Gu FM, et al. Overexpression of CD151 as an adverse marker for intrahepatic cholangiocarcinoma patients. Cancer. 2010; 116: 5440-51.

8. Blumenschein GR, Jr., Mills GB, Gonzalez-Angulo AM. Targeting the hepatocyte growth factor-cMET axis in cancer therapy. Journal of clinical oncology : official journal of the American Society of Clinical Oncology. 2012; 30: $3287-96$.

9. Zhang YW, Su Y, Volpert OV, Vande Woude GF. Hepatocyte growth factor/scatter factor mediates angiogenesis through positive VEGF and negative thrombospondin 1 regulation. Proceedings of the National Academy of Sciences of the United States of America. 2003; 100: 12718-23.

10. Garcia S, Dales JP, Charafe-Jauffret E, Carpentier-Meunier S, Andrac-Meyer L, Jacquemier J, et al. Overexpression of c-Met and of the transducers PI3K, FAK and JAK in breast carcinomas correlates with shorter survival and neoangiogenesis. International journal of oncology. 2007; 31: 49-58.

11. Drebber U, Baldus SE, Nolden B, Grass G, Bollschweiler E, Dienes HP, et al. The overexpression of c-met as a prognostic indicator for gastric carcinoma compared to p53 and p21 nuclear accumulation. Oncology reports. 2008; 19: 1477-83.

12. Lutterbach B, Zeng Q, Davis LJ, Hatch H, Hang G, Kohl NE, et al. Lung cancer cell lines harboring MET gene amplification are dependent on Met for growth and survival. Cancer research. 2007; 67: 2081-8.

13. Nakamura Y, Niki T, Goto A, Morikawa T, Miyazawa K, Nakajima J, et al. c-Met activation in lung adenocarcinoma tissues: an immunohistochemical analysis. Cancer science. 2007; 98: 1006-13.

14. Boix L, Rosa JL, Ventura F, Castells A, Bruix J, Rodes J, et al. c-met mRNA overexpression in human hepatocellular carcinoma. Hepatology. 1994; 19: $88-91$.

15. Suzuki K, Hayashi N, Yamada Y, Yoshihara H, Miyamoto Y, Ito Y, et al. Expression of the c-met protooncogene in human hepatocellular carcinoma. Hepatology. 1994; 20: 1231-6.

16. Miyamoto $M$, Ojima H, Iwasaki $M$, Shimizu H, Kokubu A, Hiraoka N, et al. Prognostic significance of overexpression of c-Met oncoprotein in cholangiocarcinoma. British journal of cancer. 2011; 105: 131-8.

17. Park HJ, Kim K, Paik JH, Chie EK, Kim S, Jang JY, et al. Is c-Met oncoprotein expression an adverse prognosticator in extrahepatic bile duct cancer treated with curative resection followed by adjuvant chemoradiotherapy? Clinical \& translational oncology : official publication of the Federation of Spanish Oncology Societies and of the National Cancer Institute of Mexico. 2016; 18: 625-31.

18. Ha SY, Lee J, Kang SY, Do IG, Ahn S, Park JO, et al. MET overexpression assessed by new interpretation method predicts gene amplification and poor survival in advanced gastric carcinomas. Modern pathology : an official journal of the United States and Canadian Academy of Pathology, Inc. 2013; 26: $1632-41$

19. Endo K, Yoon BI, Pairojkul C, Demetris AJ, Sirica AE. ERBB-2 overexpression and cyclooxygenase-2 up-regulation in human cholangiocarcinoma and risk conditions. Hepatology. 2002; 36: 439-50.

20. Terada T, Nakanuma Y, Sirica AE. Immunohistochemical demonstration of MET overexpression in human intrahepatic cholangiocarcinoma and in hepatolithiasis. Human pathology. 1998; 29: 175-80.

21. Hida Y, Morita T, Fujita M, Miyasaka Y, Horita S, Fujioka Y, et al. Clinical significance of hepatocyte growth factor and c-Met expression in extrahepatic biliary tract cancers. Oncology reports. 1999; 6: 1051-6.

22. Aishima SI, Taguchi KI, Sugimachi K, Shimada M, Sugimachi K, Tsuneyoshi M. c-erbB-2 and c-Met expression relates to cholangiocarcinogenesis and progression of intrahepatic cholangiocarcinoma. Histopathology. 2002; 40: 269-78.

23. Kim ST, Do IG, Lee J, Sohn I, Kim KM, Kang WK. The NanoString-based multigene assay as a novel platform to screen EGFR, HER2, and MET in patients with advanced gastric cancer. Clinical \& translational oncology : official publication of the Federation of Spanish Oncology Societies and of the National Cancer Institute of Mexico. 2015; 17: 462-8.

24. Barat S, Bozko P, Chen X, Scholta T, Hanert F, Gotze J, et al. Targeting c-MET by LY2801653 for treatment of cholangiocarcinoma. Molecular carcinogenesis. 2016; 55: 2037-50.

25. Valle JW, Furuse J, Jitlal M, Beare S, Mizuno N, Wasan H, et al. Cisplatin and gemcitabine for advanced biliary tract cancer: a meta-analysis of two randomised trials. Annals of oncology : official journal of the European Society for Medical Oncology. 2014; 25: 391-8. 